\title{
Characteristics of Biodegradable Implants
}

\author{
Samson Oluropo Adeosun, Ganiyu Ishola Lawal, Oluwashina Philips Gbenebor* \\ Department of Metallurgical and Materials Engineering, University of Lagos, Lagos, Nigeria \\ Email: samsonoluropo@yahoo.com, gilawal@yahoo.com, ${ }^{*}$ gbeneborphilips@yahoo.co.uk
}

Received 27 December 2013; revised 29 January 2014; accepted 8 February 2014

Copyright @ 2014 by authors and Scientific Research Publishing Inc.

This work is licensed under the Creative Commons Attribution International License (CC BY).

http://creativecommons.org/licenses/by/4.0/

(c) (i) Open Access

\section{Abstract}

The development of synthetic biomaterials for bone fixations has greatly enhanced orthopedic surgery efficiency over the last two decades. With the advancement in medical technology, several materials such as metals, ceramics, polymers and composites have been considered over the years for possible implantation into the body. These materials however, must have the following required properties that will qualify them as potential medical devices: biocompatibility, mechanical properties, corrosion resistance, creep resistance, etc. The quest in making up for the disadvantages of metallic fixations has culminated in a paradigm shift to the use of biodegradable polymers. Biodegradable polymers are light-weight materials with low elastic moduli between 0.4 - 7 GPa. These materials can be engineered to degrade at rates that will slowly transfer load to the bone. In addition, complications like corrosion, release of metal ions and stress shielding associated with metal implants are eliminated. This review considers studies carried out on most commonly investigated and widely used synthetic biodegradable polymers, their successes and limitations. It also provides process for efficient utilization of these polymers as bone fixtures without inflammation and stress shielding.

\section{Keywords}

Biodegradable; Elastic Moduli; Inflammation; Orthopedics; Stress Shielding

\section{Introduction}

In orthopedic surgery, biomaterials such as metals, ceramics, polymers and composites are being considered as implants that can intimate contact with living tissues [1] [2]. Over the years, different metallic alloys have been widely used for fracture fixations owing to the good mechanical properties and biocompatibility they possess [3]. Commonly used orthopedic alloys include stainless steels, cobalt, magnesium and titanium-base alloys [4] [5]. However, it has been established that after the removal of internal metallic fixations, the residual bone still re-

\footnotetext{
*Corresponding author.
} 
mains weaker than normal [6] [7]. This has been attributed to the residual screw holes and also, to the adverse effect of rigid plates on bones which leads to the development of stress protection atrophy. This phenomenon arises as a result of huge difference in elastic modulus between the metal fixture and the bone [2].

Bone is a complex and dynamic living tissue that provides mechanical support for the body [8] [9] and exhibits elastic modulus between 10 - $30 \mathrm{GPa}$ [10]. The elastic moduli of titanium alloy $\left(\mathrm{Ti}_{6} \mathrm{Al}_{4} \mathrm{~V}\right)$, stainless steel and chrome-cobalt alloys are 120 [10] [11], 200 and 230 GPa [2] respectively. The mismatch in elastic moduli between the bone and metallic implants is not favourable for bone healing. For instance, a fractured bone fixed with a non-biodegradable stainless steel implant has a tendency for re-fracture on removal [12]. With this fixture, the bone does not carry sufficient load during the healing process because the stainless steel does that.

Another issue with the use of metallic implants is the risk of inflammation and infections on body tissues caused by their toxic corrosion products [13]-[15]. Wear (alongside corrosion) is a surface degradation process that has limited the use of metals such as titanium as biomedical devices [16]. Contact between surfaces of artificial joints could lead to the formation of particulate and soluble metallic debris that can migrate locally or systemically with inflammatory events.

The desire to make up for the disadvantages of metallic fixations has culminated in a paradigm shift to the use of biodegradable polymers. Biodegradable polymers are light-weight materials with low elastic moduli between 0.4 - $7 \mathrm{GPa}$ [17] [18]. Implants made of biodegradable polymers can be engineered to degrade at rates that will slowly transfer load to the bone [12]. In addition, complications like corrosion, release of metal ions and stress shielding associated with metal implants are eliminated [1] [17].

The most commonly investigated and widely used synthetic biodegradable polymers are polyglycolide/glycolic acid (PGA), polylactide/lactic acid (PLA) and poly- $\varepsilon$-caprolactone (PCL) [18]-[20]. Polylactide exists in two stereo isometric forms signified by L or D [12]. If the polymer consists of only the L-isomer, it is called poly-L-lactic acid or lactide, PLLA and if on the other hand, the polymer consists of only the D-isomer, it is called poly-D-lactic acid or lactide, PDLA. The combination of the two isomers is called stereo copolymer poly-D,L lactic acid or lactide (PDLLA). In the human body, L-isomer exists in carbohydrate metabolism while the D-isomer is found in acidic milk [21].

\subsection{Challenges of Biodegradable Polymers}

The general criteria for selecting a polymer for use as an orthopedic device are its ability to combine mechanical properties with degradation time to the needs of the application such that sufficient strength remains until the bone is healed. A goal of implantology study is to design devices that induce controlled, guided and rapid healing [22]. Designing an implant to slowly degrade and transfer stress to the surrounding tissue as it heals at appropriate rate, is one of the major challenges that researchers face [12]. Although, these frequently used synthetic polymers may have excellent properties, there are drawbacks when faced with requirements for certain applications as their diverse properties have engendered their differences in resorption time. The approximate time for complete resorption of PGA is between 6 - 12 months, for PCL and PLLA is beyond 36 months, while for PDLLA is between 12 - 15 months [17].

To avoid complications and failure of the surgery, it is necessary for complete degradation of polymer to match the bone-healing time. This thus requires the creation of new materials that can degrade at rates that satisfy the conditions of healing and load-carrying ability. Most researches in this area have used the combination of two or more polymers to enhance synergic and controlled characteristics. Bibber, [23] proposed that the most preferable lactide-glycolide ratio is that of $90 \%$ polylactide and $10 \%$ polyglycolide (90:10 PLGA) adaptation. This is because the polymer is easy to handle as it does not display soft and gooey feature of high composition of glycolide. In a study by Dunne, et al. [24], degradation characteristics of PLGA 50:50 can be influenced by considering factors such as processing conditions, particle features and releasing media temperature. Low and high molecular weights of PLGA 50:50 with PLGA 85:15 have been implanted in sheep and found to be nontoxic as the breakdown of their degradation products did not produce damaging inflammatory response at the end of 8 weeks of implantation [25]. A non-crystalline polymer composed of $70 \%$ poly-L-lactide and 30\% poly-D,L-lactide (70:30 PLDLLA), having a degradation time of 12 - 18 months, has been chosen by MAST Biosurgery [26] to be adequate as tissue implants. This adopted by Ciambelli, et al. [27], resulted in PDLLA 70:30 usage with improved biocompatibility. A scaffold made of 50\% PLA and 50\% PCL (PLCL 50:50) has been found to be efficient for vascular tissue engineering as it gives perfect tissue compatibility [28]. 
A careful study of the variation in the combining ratios of these polymers needs to be undertaken while considering factors that may affect their degradation. The rate at which the polymer loses its mechanical strength per exposure time can be used to evaluate the rate of load transfer to the bone. Relationship among polymer degradation parameters with blend ratio needs to be investigated for the use of appropriate polymer in bone surgery.

Moreover, a major challenge affecting the commercialization of synthetic polymers is cost [29]. To curb this, relatively abundant natural polymer like starch, a source of PLA [30] is being used in bone tissue engineering [31] to reduce the dependence on fossil fuels. In the marine environment, chitin is confirmed to be the second most abundant biopolymer in nature after cellulose [32] [33] which forms the main exoskeletons of arthropods such as crustaceans. It has been extracted from crab, crayfish, periwinkle and shrimp [34] and used as implant [35]-[38]. The degradation behaviour of chitin and its blends with the synthetic polymers and starch needs to be investigated.

In addition to experimental study, constitutive models that describe mechanical behavior during degradation have been developed and validated with experimental results [39]. These models are only valid for low thickness devices in the first steps of erosion. It is recommended that, detailed models that would consider degradation rate dependence on temperature, environment and stress state need to be updated for further complicated needs.

\subsection{Theoretical Background}

Natural and synthetic bioresorbables (use of biodegradable polymers in orthopedics) do degrade over time by chemical hydrolysis of hydrolytically unstable back bone [12] [40]. The process occurs by penetration of water into the polymer network which cleaves its bond and eventually transforms the solid polymer into a water-soluble one. Hydrolysis of solid biodegradable implants is associated with macroscopic changes of device appearance [41] [42], changes in mechanical properties [28] [39] [43] swelling [44] and weight loss [42] [45].

Two types of biodegradation have been described in literature, which are bulk and surface erosion [12] [19] [40]. Bulk erosion occurs when the rate at which water penetrates into the polymer matrix is faster than the bond cleavage rate, i.e. the rate at which the polymer is transformed to a water-soluble material. The uptake of water takes place throughout the entire volume of the solid device and culminates in cracks and fragments [40]. For instance, PGA implanted in the femoral medullary canal of a rabbit fragmented after 21 days of implantation [46]. Blend of PGA with PDLA and PLLA in the ratio 10/10/80 fragments on the 24th week after incubation in phosphate buffer solution [47]. Majority of the medical polymeric devices are found to degrade via bulk erosion [19]. In the case of surface erosion, the rate of polymer bond cleavage is more rapid than the influx of water into the polymer matrix. The erosion is therefore limited to the surface of the polymer device which becomes thinner with time while it maintains its structural integrity over many periods of degradation.

In summary, rate of the erosion process has been confirmed to be influenced by stability of polymer back bone [19], hydrophilicity/hydrophobicity of the monomer [45] [48], polymer morphology [49], initial molecular weight of polymer [50] presence of additives, catalysts or plasticizers [51]-[53], fabrication/processing technique [54]-[56], implant surface-volume ratio, i.e. porosity [57] and the geometry of the implanted device [58].

\section{Single Polymers Characterizations}

Gauser et al., [59] characterized PLLA containing 4.5 wt\% lactic acid by mass changes, gel permeation chromatography (GPC), wide and small-angle X-ray scattering (WAXS and SAXS), simultaneous SAXS with tensile testing, and visual observation for degradation. Crystallisation of samples occurred during water up-take as degradation proceeded and this decreased SAXS period by 4 days when crystal-mediated deformation mechanism occurred. This occurrence led to "peanut-lemon"-shaped SAXS pattern, interpreted in terms of cavitation and fibrillated shear. Similar deformation mechanism at different sample displacements occurred on the 12th day of degradation. Water uptake and mass loss increased between 30 and 40 days of degradation while crystallinity of samples was stabilized. They reported that design of devices can be undertaken via imposed microstructures for a tailored evolution of mechanical response.

Thermal degradation of PLA has been investigated under inert atmosphere using both isothermal and dynamic heating regimes via thermogravimetry analysis (TGA) [60]. The researchers employed two kinetic models, one for each regime to fit the experimental data. Results demonstrated that the thermal degradation of PLA is a onestage process within the range $0 \%-30 \%$ of weight loss, having activation energy of $21-23 \mathrm{~kJ} / \mathrm{mol}$. 
Nugroho and Mitomo [61] used $\gamma$-rays irradiation at $25^{\circ} \mathrm{C}$ for biodegradation evaluation and electron beam irradiation at $90^{\circ} \mathrm{C}$ for high temperature stability study of PLA. In evaluating the effects of irradiation at high temperature, the PLA sheets were prepared in several ways vis-a-viz 1) annealing at $90^{\circ} \mathrm{C}, 2$ ) irradiation at room temperature, 3) irradiation at room temperature after annealing at $90^{\circ} \mathrm{C}, 4$ ) annealing at $90^{\circ} \mathrm{C}$ after irradiation at room temperature and 5) irradiation at $90^{\circ} \mathrm{C}$. Gamma-irradiation of samples occurred both in air and vacuum at a dose rate of $10 \mathrm{kGy} / \mathrm{h}$ for various periods while dose for the electron beam sample irradiation varied by adjusting exposure time. Enzymatic degradation of PLA powder was also carried out in phosphate buffer of $\mathrm{pH} 7.4$ containing the enzyme proteinase-K for weight loss measurements. The degradation of PLA was observed to occur by a chain-scission in both atmospheres at low dose. At higher irradiation dose, the decrease in molecular weight was found to be less pronounced. It was suggested that recombination reaction of free radicals became almost equal to chain-scission reaction. In the enzymatic degradation test, weight loss values of irradiated PLA samples decreased with molecular weight declination by irradiation and the introduction of crosslinking by irradiation was said to be a reason for this. Combining annealing and irradiation at high temperature $\left(90^{\circ} \mathrm{C}\right)$ was thus confirmed to improve PLA heat stability and rearrangement of some chain scissions which ends up increasing its crystallinity.

Fracture behavior of quenched PLA sheets having different D-lactic monomer contents of $4.25 \%$ and $2 \%$, obtained by cast sheet extrusion and de-aging has been studied [62]. The study involved de-aging the D-monomer contained PLA in oven at $60^{\circ} \mathrm{C}$ for 20 minutes followed by quenching in an iced water bath. Characterization methods used include the use of a DSC, universal testing machine and the essential work of fracture (EWF). The results showed that heating at temperatures close to glass transition temperature $\left(\mathrm{T}_{\mathrm{g}}\right)$ and subsequent quenching treatment yields a de-aging effect with an increase of the free volume of polymeric chains. Quenching enhanced brittle-to ductile changes in the fracture behaviour of PLA irrespective of the D-lactic isomer content, with a decrease on the tensile strength, appearance of shear yielding and a localized neck formation. The use of the EWF method to characterize the fracture toughness of PLA enhanced the measurement on toughness after quenching and de-ageing treatments.

Effects of crystallization and loading rate on the mode I fracture toughness and mechanism of PLA studied by Park et al., [63] involved the investigation of thermal and dynamic mechanical properties of the PLA. Fracture tests with Mode I revealed crack growth behaviours and fracture surfaces under polarizing microscope (POM) and SEM. Results showed that static values of critical mode 1 energy release rate $\left(\mathrm{G}_{\mathrm{IC}}\right)$ decreased with increase in crystallinity due to the disappearance of multiple craze formation which corresponded to the decrease of amorphous region. Observations for the amorphous PLA showed that at impact loading rate, $\mathrm{G}_{\mathrm{IC}}$ increased due to increase of fibril structure. The study also indicated that the impact toughness for the crystallized PLA was superior to the static one and was attributed to the formation of fibril structure at the impact rate.

Rods of stainless steel and PLLA have been implanted into the femoral bone of young pigs for 4 and 12 weeks in other to access early and complete tissue healing [64]. Similar notable changes in chemical and mechanical properties were observed in both materials during the period of study with no significant polymer implant resorption. Reactive bone changes between screws of self-reinforced poly-L-lactide (SR-PLLA) and stainless steel fixations of the distal femoral osteotomies in rabbits between 6 and 36 weeks have been investigated [65]. With the use of plain films, quantitative computed tomography (QCT) and magnetic resonance imaging (MRI) for bone change characterization, cortical bone density on the metallic fixture significantly reduced. However, that with SR-PLLA fixture did not. In the 36th week, oedematous zones were observed to be smaller in the SR-PLLA fixation group than in the metallic one. Results of all the three imaging modalities pointed towards the same direction as each gave indirect evidence towards a more rapid and better osteotomy healing with more in the physiologically elastic SR-PLLA screws than with the metallic screws. Thus, the results of SRPLLA screws showed that SR-PLLA will prevent stress-shielding.

Effects of processing conditions which are known to change bulk properties and not composition on the surface properties of PLLA has been investigated by Park and Cima [54]. Amorphous PLLA films were produced by melting (at $200^{\circ} \mathrm{C}$ ) solvent-cast PLLA in a vacuum oven (125 Torr) followed by rapid quenching in shallow water bath. This was followed with annealing at $70^{\circ} \mathrm{C}$ in a vacuum (125 Torr) and finally at 0.010 Torr for 24 hours. The procedure yielded crystalline PLLA films which were characterized using DSC, WAXD and SEM. A greater contact angle hysteresis observed in crystalline films was proposed to be an indicator for the differences in surface energies for the two polymers despite difficulties encountered in knowing accurate nature of differences in the surface properties between amorphous and crystalline substrates by analytical techniques. From 
their findings, processing variables are important parameters to be considered when designing devices for tissue regeneration with PLLA.

Evaluations of PLA pins produced with very little crystallinity (PLLA-A) and with extensive crystallinity (PLLA-C) in vitro in a phosphate buffer solution, PBS of pH 7.4 for 6 months were made by Duek et al., [49]. These evaluations include effects of degradation on mechanical, thermal, and structural properties, as well as molar mass variation. Results show that PLLA-C pins lose their mechanical properties more quickly, although degree of crystallinity for both types of pins increases over time. Structural analyses after 10 weeks revealed that in addition to the formation of cracks, various regions show evidence of erosion, not only along the surface of the fractures, but also on the external surface of the PLLA-A rods (Figure 1(a)). PLLA-C structure revealed an increase in the number of cracks and points of erosion which explains its complete deterioration in mechanical properties (Figure 1(b)). The protracted reduction in mechanical properties of PLLA-A with time is thus proven as a consideration for their suitability in biomedical devices.

Histomorphometrical and micro radiographical examinations of PLLA implants on skeletally mature rabbits (mean weight of $3.7 \mathrm{~kg}$ ) have been operated upon in the adjacent articular cartilage and sub-chondral bone with their intact contralateral femur which served as a control for comparison [66]. Providing 36 and 48 weeks as follow-up periods for tissues to recover after implantation, bone formation was discovered to envelope the implant in all specimens. Results also revealed a greater value of the trabeculae's fractional oseoid formation surface as compared to the intact control specimen on the 36th week. The slow healing process of the articular cartilage was attributed to the long degradation time employed. Hence, large implants made of PLLA were concluded not to be fully favourable for implantation.

Investigation on biocompatibility improvement of fast degrading viscous bioerodible polyortho ester (POE) in rabbits through addition of additives comprising sodium acetate, hydroxyapatite, calcium carbonate and magnesium hydroxide has been studied by Zignani, et al. [51]. The POE was subconjuctivally injected nasally near the superior rectus muscle with a hydraulic syringe. Daily clinical and histological examination of these restrained rabbits showed a decrease in the rate of POE degradation, which led to a reduced formation rate of acidic products. Hydrophilic sodium acetate enhanced biocompatibility modification and drug release of the polymer while magnesium hydroxide retarded drug release and improved biocompatibility. From the study, no severe inflammations occurred despite prolonged degradation rate.

Mechanical property, molecular and mass changes with in vitro degradation behavior of braided stents made of self-reinforced PLLA fibers having different diameters in PBS of $\mathrm{pH} 7.4$ and $37^{\circ} \mathrm{C}$ for 36 weeks was carried out by Pekkanuutinen et al., [67]. The mechanical properties of the stents were measured by placing each of them in the grips of the testing device at a pulling speed of $50 \mathrm{~mm} / \mathrm{min}$ and the initial radial pressure stiffness from their load-displacement curves were obtained. Initially, the radial pressure stiffness of the braided PLLA stents were found to be slightly lower than that of the commercially available metallic braided stents and it took 30 weeks for the radial pressure stiffness to decrease to half its initial value. Stents lost their structural integrity within 36 weeks during in vitro degradation. During the period, the intrinsic viscosity decreased to about half of its initial value after sterilization, while stent mass reduced to $93 \%$ of its initial value.

Barbant et al. [55] immersed dense and 90\% porous poly-L-lactic acid (PLLA) membranes in an alkaline $(\mathrm{NaOH})$ medium and PBS of $\mathrm{pH} 7.4$ and $37^{\circ} \mathrm{C}$ for in vitro degradation studies within 150 days. Results from

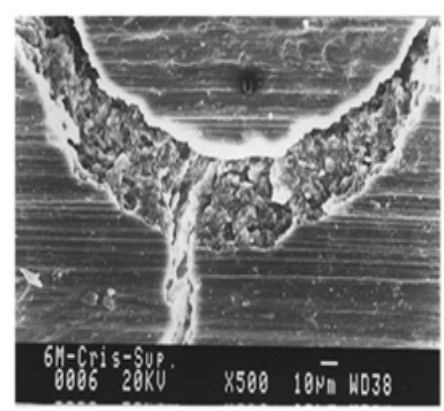

(a)

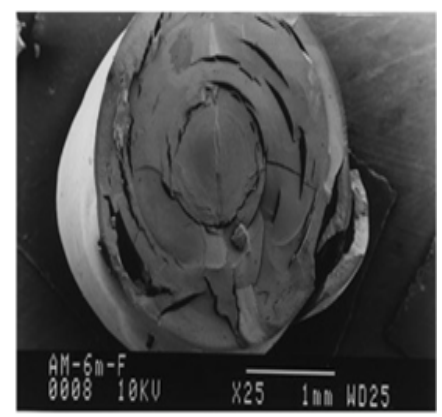

(b)

Figure 1. Surface of fracture micrographs of (a) PLLA-C and (b) PLLA-A on the 24th week [49]. 
DSC revealed that degree of crystallinity of PLLA increases with increasing degradation time in both PBS and $\mathrm{NaOH}$ but more prominent in $\mathrm{NaOH}$ medium. The study showed that pore size has no effect on the morphology of membranes during the degradation period in PBS and dense membranes degrade faster than porous PLLA by the autocatalytic effect.

In polymer tensile strength-bioerosion relationship study, Manzi [29] submerged dog-bone shaped bars of PLLA in water for 20 days. Dimensions and strength of some sets of bars till the incubation period elapsed were taken at an interval of 5 days. The results indicated that tensile strengths of PLLA do not show significant decrease with increasing exposure time in water while the fatigue time before failure is inversely proportional to the time of exposure. This has been useful in determining whether bioresorbable tensile bars would be adequate replacement for titanium fixation systems. Owing to the minimal strength decrease of bioresorbables, they can thus be appropriate for bone fixation.

Comparison between in vitro degradation of porous scaffolds (75\% porosity) and non-porous thin films made of PLLA biopolymers (Figure 2) in PBS of pH 7.4 have been studied by Sultana and Abdul-Kadir [57]. Water uptake of PLLA porous scaffolds was observed to be much more than thin films. Fragmentation and scission of chains caused by porosity led to accelerated weight loss of scaffolds after 6 weeks. This was proven to dominate the degradation during the incubation period.

The influence of hydroxyl radicals $\left(\mathrm{HO}^{-}\right)$on degradation of amorphous PDLA has been studied [52]. The $\mathrm{HO}^{-}$radicals were formed by the addition of hydrogen peroxide $\left(\mathrm{H}_{2} \mathrm{O}_{2}\right)$ with ferrous sulphate $\left(\mathrm{FeSO}_{4}\right)$ and cobalt nitrate $\left(\mathrm{Co}\left(\mathrm{NO}_{3}\right)_{2}\right)$ at $\mathrm{pH} 6$ - 7 in distilled water. Immersion of specimens in the aqueous solutions and distilled water (as control) for 30 weeks, resulted in a sharp decrease in weight loss of specimens in the former medium than the latter. It was observed that chemical hydrolysis of distilled water was responsible for the hydrolytic degradation in the ester groups of PDLA control specimens, while the existence of $\mathrm{HO}^{-}$in the hydroxyl aqueous solution showed that PDLA undergoes both hydrolytic and free radical degradation. It was therefore inferred that $\mathrm{HO}^{-}$radical can influence the hydrolytic degradation of PDLA. In a comparative in vitro degradation study, Grizzi et al. [58] aged varying dimensions and shapes of poly-D,L-lactic acid (PDLA) devices in phosphate buffer solution (PBS) of $\mathrm{pH} 7.4$ at $37^{\circ} \mathrm{C}$ for a maximum of 36 weeks. Samples with greater thickness degraded at a faster rate and therefore, nanoparticles should last much longer than large size devices for effective use in drug transport and sustained release. Schmidmaier et al. [53] coated titanium and steel wires with PDLA containing growth factors that will stimulate fracture healing. The growth factors include insulin-like growth factor-I (IGF-I, 5\% W/w) and transforming growth factor-beta 1 (TGF- $\beta 1,1 \% \mathrm{~W} / \mathrm{w}$ ). A 42 day in vivo degradation experiment in tibiae of rats revealed a loss of PDLA thickness (less than 5\%) with a complete and regular layer without any defect on the metallic surfaces. The results confirmed that growth factors can be released continuously from implants directly into the bone (especially at the early phase of bone healing) to stimulate healing and in-growth processes. The ability to influence the proliferation and differentiation of cells was said to enhance this stimulation. An experiment aimed at investigating the relationship between oligomer molecular weight and in vitro degradation of PDLA in $65^{\circ} \mathrm{C}$ PBS of $\mathrm{pH} 1.5,4.5$ and 7.4 has been carried out by Schliecker, et al. [41]. The polymers were characterized via Size Exclusion Chromatography (SEC), DSC and Neutron Magnetic Resonance (NMR). Results from kinetic studies revealed that hydrolytic degradation of oligomers at $\mathrm{pH} 1.5$ was the fastest, while that of $\mathrm{pH} 4.5$ is the slowest. From the mechanistic point of view with

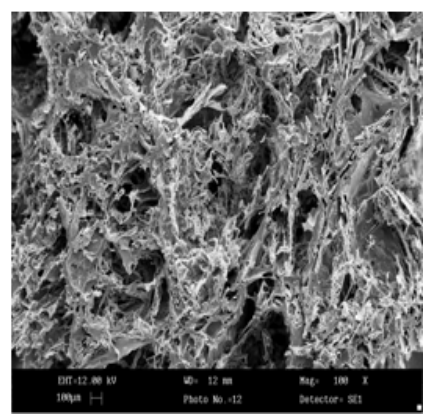

(a)

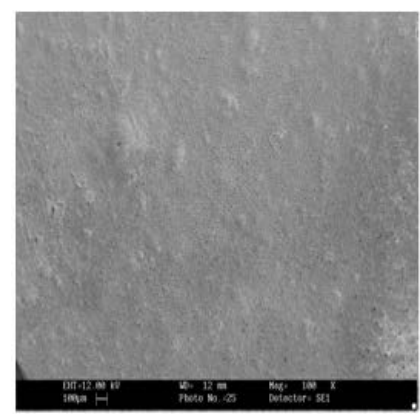

(b)

Figure 2. Scanning electron micrograph (SEM) of PLLA (a) porous scaffold (b) thin film [58]. 
H-NMR spectroscopy, chain end cleavage was said to be responsible for the degradation of oligomers in the acidic medium ( $\mathrm{pH}$ 1.5) while for basic, degradation was attributed to random ester cleavage. Thus, hydrolysis is said to slow down with decreasing $\mathrm{pH}$ up to $\mathrm{pH} 4.5$ but faster below 4.5. The study also showed that degradation at the initial stage deviates from the conventional autocatalytic effect. Further studies on factors responsible for bulk degradation of PLA and PLGA was advocated.

Two materials have been used to perform osteotomy on the medial femoral condyle of sheep: PDLA pins and composite pins of $10 \% \beta$-tricalcium phosphate $(\beta$-TCP) (90/10) [68]. At the end of 3, 18 and 36 months, a histological examination performed on the synovial membrane and lymph nodes revealed that minor reactions of the synovial membrane took place in the composite pins at the end of the 18th month. Owing to the slow degradation process of biodegradable PDLA, there was no clinically relevant inflammation of either joint or lymph nodes. The addition of $10 \%$ b-TCP did not result in any significant enhancement.

Kumta et al. [46] implanted rods of PGA into the femoral medullary canal (bone) and the subcutaneous tissues on the inner section of each thigh of adult rabbits. Bend tests conducted after 4, 7, 10, 14 and 21 days of implantation showed that PGA displayed 73\% strength reduction in bone implantation and 64\% reduction in subcutaneous tissue at the end of 14th day. Fragmentation of PGA rods occurred after 21 days which made it difficult to run tests on retrieved samples after implantation. An understanding of the degradation mechanism of tyrosine-derived polycarbonates from the hydrolysis rate of carbonate and ester bonds in the polymer compounds have been studied [48]. A model was designed to simulate repeat unit of tyrosine-derived polycarbonates, the degradable ester and carbonate linkages present in the pendent chain and the backbone. Incubations at $37^{\circ} \mathrm{C}$ in a $5 \mathrm{ml}$ PBS at $\mathrm{pH} 1,3,5$ and 7.4 indicated that increasing the pendent chain length from ethyl to octyl increased the hydrophobicity of the model compound and lowered the hydrolysis rate of both the ester and the carbonate bond. At neutral to slightly acidic $\mathrm{pH}$, hydrolysis rate of the carbonate bond was more rapid than the rate of ester bond cleavage. Under acidic conditions, the acid-catalyzed hydrolysis of the ester bond became a dominant factor and pendent chain ester hydrolysis outpaced the rate of hydrolysis of the backbone carbonate bonds. These observed results were affirmed to be useful in the speculation of in vitro degradation behavior of polycarbonates.

Schantz et al. [69] implanted PCL, having a porosity of approximately 70\% in patients' calvarium for clinical evaluations for 3, 6 and 12 months. This short term evaluation showed that PCL is suitable for bone temporary supporting device as no complication was observed. Their strength and fracture-resistant properties enhanced firm anchoring in the surrounding calvarium, which led to stable tissue reconstruction.

Investigation into the role of esterolytic enzymes and reactive oxygen species secreted by macrophages on the degradation of aliphatic and lysine-derived poly (ester urethane)s (PEUR) networks under both in vitro and in vivo conditions has been conducted by Hafeman et al. [70]. PEUR scaffolds were synthesized by Hexamethylene diisocyanate trimer (HDIT) or lysine triisocyanate (LTI) methods. Results showed that during the first 8 weeks of in vitro incubation, all the PEUR materials retained at least $80 \%$ of their original mass. Compressive and tensile properties of the scaffolds showed that they were compressed to $50 \%$ of their initial thickness without fracturing. The tensile strain at break ranged from $60 \%-240 \%$ for all samples. Images from SEM revealed surface degradation, thinning, and pitting of the pore walls as predominant degradation mechanisms in 4 to 8 weeks while pitting, cracking, and subsequent failure of pore walls were observed at 12 weeks.

\section{Characterizations of Co-Polymers/Polymer Blends}

Mechanical behavior of poly-D,L-lactic acid/poly-e-caprolactone (PLA-PCL, with a 90:10 ratio of PLA and $\mathrm{PCL}$ ) fibers during degradation in $37^{\circ} \mathrm{C}$ PBS have been examined [39]. At the end of 28 week incubation period, fibers' dimensions and degradation medium showed no significant change. Force-extension relationship measured from the universal mechanical testing machine revealed that plasticity and strength decreased progressively at the end of the 16th week. Constitutive models used validated experimental results and thus found to be reasonable for other biodegradable materials. This model was considered as fundamental tools in the design of regenerative medical devices where strain energy is an important requirement. Dunne et al. [24] ran an in vitro degradation test on micro and nano particles of polylactide-co-glycolide (50:50 PLGA) in phosphate saline (PBS $\mathrm{pH}$ 7.4) under different conditions of temperature. Weight loss observation of samples after incubation period showed the existence of a linear relationship between the degradation rate and particle size, with the larger particles degrading faster. The rate of polymer degradation was also found to increase with increasing incubation 
temperature. The ability to model and predict the mechanical properties of Polyglyconate B (a block co-polymer of glycolic acid and trimethylene carbonate) as a function of implantation time, have been demonstrated by Farrar and Gillson [71]. In the study, two different molecular weights of the material were incubated in PBS at $37^{\circ} \mathrm{C}$ for 31 days. Changes in molecular weight, mechanical properties and mass as a function of degradation time were observed. The polymer with lower molecular weight lost its strength faster as compared to the other. A relationship between mechanical strength and average molecular weight $\left(M_{n}\right)$ number was seen feasible while that for weight average molecular weight $\left(\mathrm{M}_{\mathrm{w}}\right)$ was not. This observed relationship was seen to be more complex than that used in the model. As a result, empirically derived model from data was suitable in determining the relationship between $\mathrm{M}_{\mathrm{n}}$ and mechanical strength of the polymer.

Varying mass fraction of PLA-PCL blends have been cast to dog-bone shapes by Broz, et al. [72] for structural and mechanical investigations using a $10 \mathrm{~mm}$ extensometer. The strain at failure measured across the entire mass fraction range of samples was observed to decrease monotonically with the mass fraction increase of PLA (Figure 3). Supporting this with results obtained from SEM and NMR, the decrease showed that little amounts of glassy PLA will embrittle the PCL matrix as a result of the formation of PLA inclusions in the blend with some interfacial adhesion. Thus, the range of mechanical properties accessed in the experiments demonstrated the benefit of polymer blending as mechanical properties can be tailored through blend composition. PLA and PCL were confirmed to be miscible and some adhesion may occur at the PLA/PCL interface when the majority phase is PCL but not the reverse.

Elastic poly-L-lactide-co-e-caprolactone (PLCL, of 50:50 PLA:PCL) scaffolds have been seeded with smooth muscle cells (SMCs) and implanted in nude mice for biocompatibility and in vivo degradation behavior studies [28]. From the study, PLA-PCL scaffolds of this ratio exhibited excellent tissue biocompatibility to SMCs, which suggested its suitability for vascular tissue engineering. It was also observed that PLCL scaffolds display slow degradation time, while caprolactone units degrade faster than lactide. This was attributed to the fact that the amorphous regions contain caprolactone moieties which degrade earlier than hard domains (crystalline) where most of lactide units are located. Thus, scaffolds' perfect tissue compatibility to SMCs permits them for vascular tissue engineering.

Blends of PLLa and PCL (PLLA/PCL), having different PCL contents of 5, 10 and $15 \mathrm{wt} \%$ have been fabricated to investigate the effects of PCL content on the fracture property, mechanism and microstructure of PLLA/ PCL [73]. Mode I fracture testing measured the critical energy release rate at crack initiation $\left(\mathrm{G}_{\text {th }}\right)$ while polarizing optical microscopy POM and SEM characterized fracture mechanisms. It was shown that $\mathrm{G}_{\mathrm{th}}$ was improved by blending PCL with PLLA and the maximum increase (51\%) was achieved with 5 wt\% of PCL.

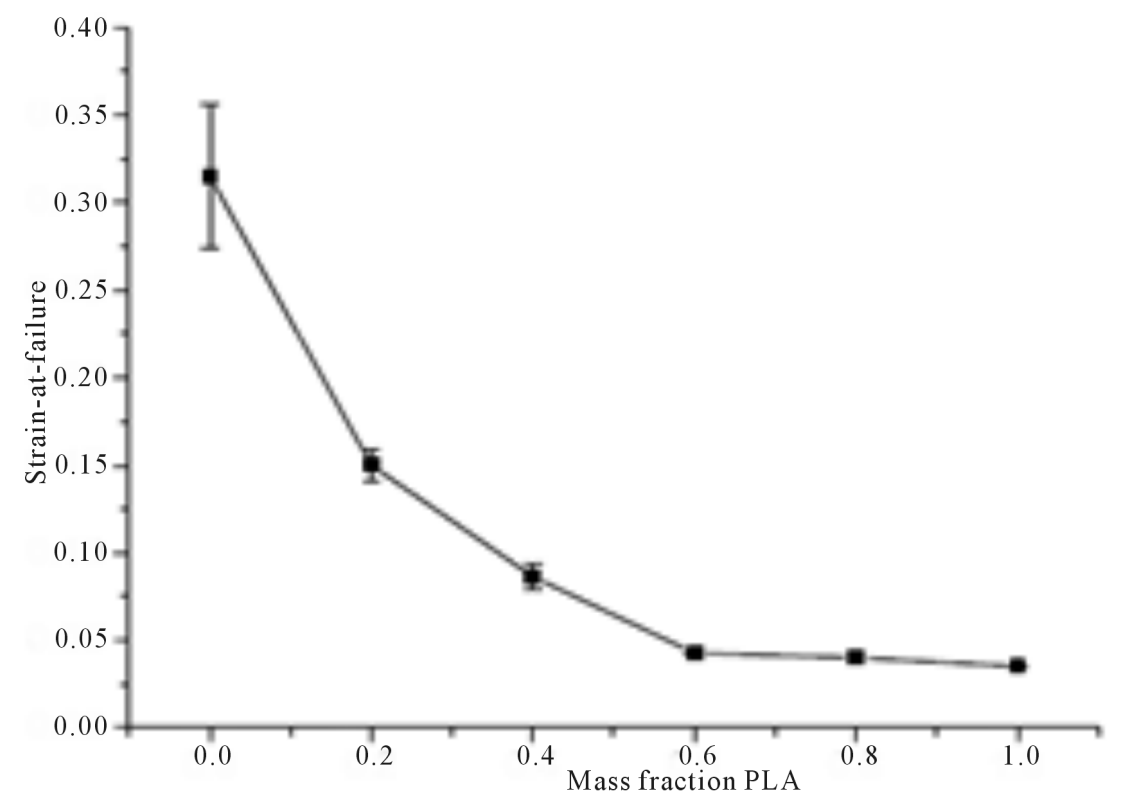

Figure 3. Monotonic decrease of strain at failure with PLA fraction increase in PLA/ PCL blends [72]. 
Morphological study showed that phase separation took place due to the incompatibility of the two components, and spherical structures were created as dispersed PCL phases. The size of PCL phases increased with increase of PCL content. PLLA/PCL exhibited craze formation in crack-tip region that was initiated by void formation and nucleated by de-bonding of spherulite interfaces. Increase of dissipated energy due to such multiple craze formation was confirmed to be the primary mechanisms of the $G_{\text {in }}$ improvement.

Salgado et al. [74] blended 5\% of a rapid degradation rate sebacic acid (SA) with PCL, known for its low degradation kinetics for degradation studies in PBS for 30, 60, 90 and 120 days. Characterization of specimen's stability evaluation was done through differential scanning calorimetry, dynamical mechanical analysis and scanning electronic microscopy after each stage of degradation. Results showed that porosity of blend increases with time as polymer crystallinity gradually decreases. Both pure PCL and PCL-SA became stiffer with the increase in time of incubation in PBS solution. A distinct behaviour between degraded and non-degraded specimens was observed on the 30th day, with the undegraded specimens having higher storage modulus with the increase in temperature. Cell culture studies on MG63 osteoblasts also showed that the PCL-SA gel is cytocompatible and capable of maintaining high cell viability. In vitro degradation studies on cylindrical macroporous polylactide-co-glycolide (PLGA 75/25) foams in sodium cacodylate buffer solutions having different $\mathrm{pH}$ values (5.0, 6.4 and 7.4) has been carried out by Holy et al. [75]. Degradation measurements through changes in mass, molecular weight, and morphology showed that foams maintained at $\mathrm{pH} 6.4$ and 7.4 lost $30 \%$ of their initial weight while that maintained at $\mathrm{pH} 5.0$ loses $90 \%$ of its initial weight in the 4th month. This implied that the foams at $\mathrm{pH}$ of 6.4 and 7.4 can be used in tissue engineering applications for tissues that regenerate within the same time frame.

Elst, et al. [76] stated that the volume quantity of polymeric implant in the bony tissue needs to be reduced to avoid severe foreign body responses. PLA, 20:80 PLA/PGA and stainless steel were implanted into the mid shaft osteotomy of the left femur of 21 female sheep so as to evaluate tissue reaction on the implants and their long term degradation after 30 months with the use of light microscopy. A total of 18 animals survived the 30-month implantation period: six animals with a stainless-steel implant, five animals with a PLA implant and seven animals with a copolymer implant. Histological reports after 30 months of implantation revealed that the degradation of the materials was lower compared to the maximum suggested period of 24 months. Furthermore, for this long term period, tissue response was found severe with these materials and this indicates repulsion risks. Different formulations of PLGA foams have been used randomly on different ribs of sheep for 8weeks by Thomson et al. [25]. The synthetic polymer scaffolds make-up were PLGA with 85:15 copolymer ratios (PLGA 85:15), PLGA with a 50:50 copolymer ratio of high weight average molecular weight (High MW PLGA 50:50) and PLGA 50: 50 of low weight average molecular weight (Low MW PLGA 50:50). Histologic analysis showed no observable evidence of significant inflammatory reaction or tissue damage from degradation products during the period of implantation. Thus PLGA 85:15 with High and Low MW PLGA 50:50 are non-toxic when implanted in sheep and breakdown products do not induce damaging inflammatory response after 8 weeks.

Biodegradation behavior and the factors affecting biodegradation rate such as polymer composition, molecular weight, and $\mathrm{pH}$ of the biodegradation media is studied by $\mathrm{Wu}$ and Weng, [50]. A series of PLGA polymer rods of similar molecular weight but varied compositions such as PLGA50/50, PLGA65/35, PLGA75/25, PLGA85/15, and PLGA100/0, were immersed in $7 \mathrm{ml} \mathrm{PBS} \mathrm{of} \mathrm{pH} 7.4$ and at $37^{\circ} \mathrm{C}$. As regards molecular weight effect on biodegradation, series of polymers with the same composition (PLGA 75/25) but different molecular weights were immersed in $7 \mathrm{ml}$ of PBS of $\mathrm{pH} 7.4$ and $57^{\circ} \mathrm{C}$. Polymer samples of PLGA 50/50 were used in the study of $\mathrm{pH}$ effects. Here, samples were immersed in PBS of $\mathrm{pH} 5.0$ and 7.4 while $\mathrm{pH} 9.24$ sodium bioatebuffer was also used. The study results indicated that PLGA with a higher molecular weight degrades faster than those with a lower molecular weight. Furthermore, the biodegradation rate of PLGA containing higher content of lactic acid moiety is lower than those containing a lower content of lactic acid moiety. Effect of medium $\mathrm{pH}$ showed that biodegradation of PLGA could be delayed in a basic medium ( $\mathrm{pH} 9.24$ ) compared to samples in an acidic medium (pH 5.0) with higher biodegradation rate. Biocompatibility of bioresorbable PLGA 85/15 pins implanted in the interstices of dorsal muscle, the gluteal muscle and the bone marrow cavity of distal femur of adult rabbits (3 and $4 \mathrm{~kg}$ ) has been studied by Lin et al. [77]. Macroscopic and histological examinations within two months after implantation revealed that the heterogeneous degradation of PLGA specimens in vivo led to erosion from the surface towards the center of the pins due to acid secretions of inflammatory cells adjacent to the implants. The accumulation of acids facilitated the cleavage of ester bonds of PLGA and this accelerated the degradation rate. Unlike center degradation pattern (interior to exterior) observed in vitro by other researchers, 
slow removal of the acid produced from the hydrolysis of polyester in the interior of the specimen was confirmed to be the cause. Inflammatory reaction was not found to be severe and healing of the surgical wound took place without complications. PLGA was therefore seen as a good bioresorbable material for medical use. Differences in degradation patterns observed in vivo and in vitro implied that regular incubation conditions proposed for in vitro study will not perfectly simulate in vivo degradation of implants. The study aimed at ensuring dimensional stability of bioresoebable polymers during degradation for suitable bone bearing capacity during application has been carried out [44]. Polylactide-co-glycolide (PLGA of 85:15 PLA:PGA) was reinforced with 3D network (scaffold) biodegradable polymer polypropylene fumarate (PPF) and crosslinked with a biocompatible hydrophilic and vinyl monomer, vinyl pyrolidone (VP). Implantation of extruded rods into $400 \mathrm{~g}$ adult male Sprague served as the in vivo model while for the in vitro, incubation of specimens was done in lead buffer solution at $37^{\circ} \mathrm{C}$ for 21 days to measure their degree of swelling, hardness and flexural strengths. Investigations showed that unreinforced PLGA possess higher initial flexural strengths than the in situ cross linked or blended samples before incubation in the buffer solution. Loss of flexural strength was evident in the reinforced samples. Substantial amount of newly formed uncrosslinked leachable PVP (polyvinylpyrolidone) leaving the implant structure was affirmed to be responsible for the decrease in crystallinity of the bulk PLGA which eventually led to decrease in its strength. The introduction of reinforcing scaffold enhanced the production of leachables which in turn influenced the degree of biological response. The in situ cross linked samples appeared to be the most suitable tissue damage. This formed procedural basis for in vivo biocompatibility.

Poly (lactide-co-glycolide) (PLGA 50/50) has been blended with hydrophilic chitin to form chin/PLGA blend ratios of 1:3, 1:2, 1:1, 2:1 and 3:1, respectively [45]. Information from SEM revealed that PLGA particulates are homogeneously dispersed in chitin matrix and this suggested the occurrence of obvious phase separation from the blended chitin and PLGA 50/50 phase which occurred as a result of their thermodynamic incompatibility. Degradation study achieved by determining water uptake capacity in deionized water at room temperature showed that weight loss of the chitin/PLGA 50/50 microspheres increase with increase in hydrophilic chitin content. This implied that the hydrolysis of PLGA can be hastened after blending with hydrophilic chitn. Temperature dependence of elastic (G') and viscous moduli (G”) on 75:25 PLA/PGA has been studied by Santovena et al. [42]. Implants were observed to behave as visco-elastic solids at room temperature, where values of G' were above that of G". Implants had high flexibility and capacity to maintain their initial shapes after deformation. On incubating samples in phosphate buffer solution (PBS) of pH 2.0 and 7.4 for 22 days, increase in G' and G” was observed, implying increase in their rigidity and flexibility. In addition, continuous weight loss and implants' appearance were observed. Degradation at $\mathrm{pH} 2.0$ was faster than that at 7.4 and this was attributed to the fact that carboxylic end-groups of polymers were not neutralized in the former. In vitro and in vivo degradation in phosphate buffer solution and rats respectively, with biocompatibility comparison between polypolyolsebacate (PPS) and PLGA have been carried out [78]. In vivo degradation results were observed to be faster than those of in vitro. In addition, the chemical, physical and mechanical properties with degradation rates of PPS can be altered by altering the polyol and stoichiometry of the reacting sebacic acid. PPS polymers exhibit comparable biocompatibility with PLGA.

Cylindrical shaped composite materials composed of $84 \mathrm{wt} \%$ lactide and $16 \mathrm{wt} \%$ glycolide (PLGA) with 15 wt\% carbon fibers and hydroxyapatite particles (HA) incubated in a Ringer solution and simulated body fluid (SBF) at $37^{\circ} \mathrm{C}$ for 10 weeks to determine environmental influence on degradation was examined [79]. The low viscosity of Ringer fluid activated rapid degradation of the composite implant than in SBF. Thus, degradation speed is dependent on viscosity of applied fluids which provides the ability to penetrate polymer's structure and interfacial boundaries. Comparing results with in vivo experiments on sub maxilla bone of rabbits, degradation process in vivo was observed to be faster than in vitro. Complexity of the living system, where processes like protein adsorption, cellular reactions, body fluid flow and charge exchanges justified the reason for such rapid degradation of artificial implants. Failure studies of 70:30 poly-L-lactide-co-D,L-lactide (PLDLLA) spinal cages designed to withstand mechanical loads in the lumbar spine of a goat for six months has been carried out by Smit et al. [80]. A servo-hydraulic MTS Elastomer Testing System 831 applied uniaxial compression load within the rates of $10^{-3}$ and $10^{-1} \mathrm{~mm} / \mathrm{s}$ to failure under standard conditions at room and body temperatures. The experiments were done in de-mineralized water after soaking for 24 hours and the effect of humidity evaluated. Observation indicated that at lower loading rates, humidity and temperature, the mechanical strength of 70/30 PLDLLA cage is low. On static loading, cages fail within 24 hours when loaded at about half their mechanical strength while prediction via extrapolation evaluated cage failure in the 3rd month when loaded at a quarter of 
their strength. Loading below their instantaneous strengths, the cages were observed to fail after short periods of time. The premature failure of 70/30 PLDLLA cages arose as a result of mechanical loading and the time-dependent mechanical properties of the material. These induced a state of enhanced molecular mobility that ended up stimulating a dynamic rearrangement of molecular segments and thus engendering a plastic flow.

The potential of 70:30 PLDLLA membranes as temporary implant under hydrolytic degradation in a PBS of $\mathrm{pH} 7.4$ at $37^{\circ} \mathrm{C}$ for 20 weeks has been studied [81]. The characterization of membranes before and after degradation were achieved using differential DSC, GPC, TGA, SEM and mechanical traction tests. Stability of membranes was observed to span for 16 weeks until noticeable decrease in $T_{\mathrm{g}}$ was observed in the 18th and 20th weeks. This was attributed to the polymeric chain cleavage that occurred during degradation. With the GPC, decrease in $\mathrm{M}_{\mathrm{w}}$ was observed after 2 weeks and the results showed that $\mathrm{M}_{\mathrm{w}}$ loss occurred between the 18th and 20th weeks as a result of gradual breakage of polymer chains during hydrolysis. Analysis with TGA showed that the temperature at which mass loss begins decreases with degradation time as its low values were recorded in the 18th and 20th weeks. Microscopic observation indicated that no pore was present before degradation but after degradation, pores and fissures on broken surface were evident. Membranes' resistance to traction and modulus of elasticity were found to be maintained till the 8th week. At the end of the 16th week, there was a sharp reduction of these properties as a result of PLDLA degradation. Given these degradation characteristics, PLDLA membranes were said to be good for temporary implantations.

In vitro degradation of 70:30 PLDLLA in PBS for 24 weeks and medical incision in dorsal subcutaneous tissue rats for 90 days have been studied by Ciambelli et al. [27]. The implants showed no effect on the health and behaviour of the rats as no significant adherence between tissue and implants occurred within the first 7 days. In contrast, 15-day implants showed greater polymer-tissue attachment. This finding suggested that the polymertissue process tends to increase gradually through organization of the conjunctive tissue fibers into a conjunctive capsule. Mechanical tension test results showed that PLDLLA behaves like a ductile material under constant tension. The Young's modulus, elongation at break and the maximal tension decrease as degradation time elapses. Polymers retained their dense morphology within the first 4 weeks of incubation until after the 12th week where pores and cracks are observed at the center of samples. Biocompatibility and mechanical stability of PLDLLA displayed in the experiments have made them attractive materials in the biomedical fields.

The effects medium $\mathrm{pH}$, filler material ( $\beta$-tricalcium phosphate. $\beta$-TCP) and crosslinking density of polypropylene fumarate-diacrylate (PPF-DA) on in vitro degradation of poly (propylene fumarate) (PPF) as an injectable and biodegradable bone cement has been examined [82]. The study employed a 52 week incubation period with the use of buffered saline having $\mathrm{pH} 7.4$ and 5.0. Within the first 12 weeks of immersion, the $\beta$-TCP composite specimens showed initial increase in their modulus and yield strengths. Lower crosslinking densities of PPF/PPF-DA networks indicated rapid degradation rate owing to the occurrence of a huge mass loss. At the lower environmental $\mathrm{pH}$ 5.0, degradation was hastened but this effect was not as intensified as that of crosslinking density due to low water uptake of the material. In comparison to the physiological $\mathrm{pH} 7.4$, rapid decrease in the compressive strength at $\mathrm{pH} 5.0$ with no difference in mass loss occurred. The addition of $\beta$-TCP into the polymer matrix acted as an internal buffer as degradation was delayed. Results showed that the degradation of PPF/PPF-DA networks can be controlled by the crosslinking density, accelerated at a lower $\mathrm{pH}$, and prolonged with the incorporation of the b-TCP filler.

Glarner and Gogolewski [47] studied in vitro degradation of poly-L-lactide-co-DL-lactide-co-glycolde (80/ 10/10 PLLA/PDLA/PGA) pins in PBS of $\mathrm{pH} 7.4$ and $37^{\circ} \mathrm{C}$ for 36 weeks. Results showed a progressive drop in molecular weight and mechanical properties of pins until the 24th week where fragmentation occurred. Crystallinity of pins increased during the first few weeks of incubation but declined on the 24th week. The high susceptibility of the pins to in vitro degradation was associated with the chemical composition and the molecular weights of samples used in the study. Toto et al. [83] blended four different kinds of ductile biodegradable polymers with PLLA to study its effects on the bending modulus, strength, impact fracture energy and mode I fracture property. These polymers were PCL, polybutylenessuccinate (PBS), polybutylene succinate-co-L-lactate (PBSL) and polybutylenessuccinate-co- $\varepsilon$-caprolactone (PBSC). A field emission scanning electron microscope (FE-SEM) investigated mode I fracture while a POM measured the crack growth behaviour. Though ductility of PBS was thought to be lower than the other three ductile polymers, blending it with PLLA improved its modulus, strength and toughness. Thus, polymer with the least ductility was concluded an effective blending partner to improve the bending properties of PLLA. PLLA/PBSC exhibited the best fracture properties such as mode I fracture energy and impact perforation energy. Ductility of PBSC was the highest and therefore, loca- 
lized ductile deformation in crack-tip region was effectively enhanced. Fibers of PLA-PCL, PGA-PCL, PDO and PGA have been characterized in terms of their degradation rates in water, $\mathrm{NaCl}$ (saline solution) and PBS at $37^{\circ} \mathrm{C}$ [43]. Although the degradation rate of PCL is naturally very slow, weight loss results of the PGA-PCL combination in the three media is the most rapid. This is followed by PGA, PDO and the least degradable, PLAPCL. PBS was observed to be a fast degrading medium for polymers except for PDO where water with $\mathrm{pH}$ nearly neutral degrades much faster than the other two media. No significant differences were observed among the three media for PLA-PCL. At the end of each degradation stage, mechanical test results showed that tensile strength of the samples depend on molecular weight while the Young modulus is independent of it. This degradation rate can be used in the dimensioning of biodegradable devices using a first order equation. In cases where diffusion is neglected, the rule of mixtures could be used to calculate the degradation rate of a composite of two or more biodegradable materials.

Schakenraad et al. [84] studied the in vivo and in vitro degradation of glycine/D,L-lactic acid copolymers with poly-D,L-lactic acid in rats and PBS at pH 7.4 respectively. The degradation of the polymers was observed to take place via bulk hydrolysis of the ester bonds and they showed comparable in vivo and in vitro degradation behaviour. Tissue reactions against all materials started with an acute inflammatory reaction caused by the trauma of implantation and followed by wound healing processes. These results in mild foreign body reaction for the poly-D,L-lactic acid and a more excessive macrophage mediated foreign body reaction for the glycine/ DL-lactic acid copolymers. Although the rate of hydrolysis was not affected by the changes in composition, increasing the mole fraction of glycine units in the copolymer promotes faster absorption of material (a higher degradation rate) which makes tissue reaction more severe. Riley et al. [85] evaluated effects of polymer chemistry on adhesion, proliferation, and morphology of HAC. The evaluation studies was done on the following polymer films: PGA, PLLA, PDLA, 85:15 PDLA-PLGA, PCL, 90:10 PDLA-PLCL, 9:91 PDLA-PLCL, 40:60 PLLAPLCL, 67:33 polyglycolide-co-trimethylene carbonate (PGTMC), and PDO as polymer scaffold material for human articular cartilage (HAC) and chondrocytes, harvested from the knees of a young cadaveric donor. The kinetics of cell adhesion was assessed by seeding HAC cells into tissue culture polystyrene (TCPS) and PCL substrates in a humidified incubator $\left(37^{\circ} \mathrm{C}\right.$ and $\left.5 \% \mathrm{CO}_{2}\right)$ for $0.25,0.5,1,2,4,6,8$, and 10 hours. The seeding of HAC cells onto all polymers for 8 hours was also done and cell proliferation assessed. Chondrocyte adhesion is observed to be rapid and close to its maximum within 6 hours of culture. The greatest number of chondrocyte attachment after 8 hours of culture occurred on PGA and 67:33 PGTMC polymer films, while attachment to PCL and L-PLA films were statistically lower than that found on PGA. When the chondrocytes were allowed to proliferate on the substrates, a superior expansion was observed on PDO and L-PLA substrates compared to the TCPS control. This was being partially attributed to the superior hydrophilicity of the PDO and L-PLA polymer compared to the TCPS. With respect to adhesion and proliferation, the results have shown that all polymers used in the study would provide a suitable substrate for chondrocyte culture and potential scaffolding materials for cartilage tissue engineering.

In vitro degradation studies of polymerization/copolymerization procedure of PLA a and PLGA copolymer of 90:10 and 70:30 ratios in PBS maintained at $37^{\circ} \mathrm{C}$ and $\mathrm{pH} 7.4$ has been carried out by Kiremitci and Deniz [86]. Results showed that homopolymers exhibit similar degradation rates while the copolymers degrade more rapidly depending on the glycolic ratio. Comparison of mass loss and intrinsic viscosities results showed that mass loss of implants occurred after intrinsic viscosity reached a threshold level. Self-reinforced polyglycolic acid (SRPGA) and poly-L-lactic acid (SR-PLLA) pins have been fixed into the right and left femurs of rats for their shear load carrying capacity after 1, 3, 6, 12, 24, 36, 48 and 52 weeks of implantation [87]. Shear load carrying capacity of femur devoid of implants (control sample) and those fixed with SR-PGA and SR-PLLA implants were observed to possess maximum values on the 36th week after which they gradually reduced. On the 52nd week, the load bearing influence of pin on SR-PLLA implants ceased as SR-PGA implants and control specimens had greater magnitudes of load bearing capacities. Periods other than this revealed higher load bearing capacity values in the SR-PLLA implants where pins carried the load.

Films and porous patches of poly-3-hydroxybutyrate (PHB) and PLLA have been fabricated and prepared for mechanical testing, thermal and molecular weight analysis for the development of a gastro-intestinal patch [88]. Tensile properties of specimens at room temperature are measured from the stress-strain curves, while thermal and molecular weight analysis are perfumed with the use of DSC and the GPC respectively. In vitro degradation experiment were carried out on the materials in Sorensen buffer $(0.1 \mathrm{M}, \mathrm{pH} 7.4)$, kept at $37^{\circ} \mathrm{C}$ and $70^{\circ} \mathrm{C}$ respectively. Implantation into the abdomen of $250 \mathrm{~g}$ anesthetized male rats represented the experiment in vivo. In ad- 
dition, immersion of samples in pancreatin (an enzyme) investigated enzymatic influence on the degradation. In vitro degradation results showed that hydrolysis of PHB is slow as its molecular weight loss decreases by $1.5 \%$ after a year in buffer solution at $37^{\circ} \mathrm{C}$ while the degradation of PLLA was much faster. The presence of pancreatin indicated a participation of enzymes on PHB hydrolysis, while the degradation characteristics of PLLA remained essentially the same. For repair of bowel defects, experiment showed that PHB is selected as most promising material combination because of its mechanical properties and in vitro degradation behavior. Its degradation occurs in a way that the temporary patch material resists the intestinal secretions and closed the bowel defect for a sufficiently long time to allow complete healing of the defect. Three processing stages, which are the orientation, hot-stretching and post annealing stages, have been designed for PGA and PGLA fibers for structural, property changes and in vitro degradation assessments [56]. PGA fibers showed higher degrees of crystallinity than corresponding PGLA fibers in the orientation stage while PGA samples gained more crystallinity and higher glass transition temperature, $\mathrm{T}_{\mathrm{g}}$ than PGLA samples in the hot stretching and post annealing stages. PGA samples' degradation rate was slow as a result of its high degree of crystallinity while results from dynamic mechanical analysis (DMA) indicated that the internal stress built in the orientation and hot stretching stages was released during the post annealing stage for both PGA and PGLA samples. Increase in sample strength, crystal size and $\mathrm{T}_{\mathrm{g}}$ were observed at the hot stretching and the annealing stages. Both PGA and PGLA samples showed cleavage-induced crystallization during in vitro degradation.

Degradation rates of fast degrading polymers, 50:50 PLGA and slow degrading polymer, PCL have been differentiated by Sung et al. [89]. Smooth muscle cells were harvested from aortas of wild-type mice and grown in Dulbecco/Vogt modified Eagle's minimal essential medium (DMEM) supplemented with 10\% fasting blood sugar (FBS). Observation showed that fast degradation of PLGA negatively affects cell viability and migration into the scaffold. This effect was ascribed to the significant acidification of the local environment due to the fact that polymer degradation of the PLGA scaffolds exhibit less cell mobilization and less angiogenesis which further supports the negative effect of the acidic environment created by the degradation of biocompatible polymers. Angiogenic response was developed within the scaffolds implanted in vivo and this was attributed to the presence of inflammatory response.

Two in vivo degradation experiments have been performed on segmented polyethylene glycol (PEG) and polybutylene terephthalate (PBT) (PEOT/PBT) by Deschamps et al. [90]. Subcutaneous implantation of meltpressed discs of varying copolymer composition in rats for 24 weeks marked the first in vivo experiment. In other to incite prolonged in vivo degradation, pre-degraded PEOT/PBT samples in phosphate buffer saline (PBS) at $100^{\circ} \mathrm{C}$ for 14 days were made and subsequently implanted in the rats, which represented the second experiment.

Materials in both experiments were observed to degrade slowly with the exception of polymer with the higher content of PEO, whose degradation was rapid. Degradation products in PBS as analyzed by proton nuclear magnetic resonance spectroscopy (1HNMR) and high performance liquid chromatography/mass spectroscopy (HPLC/MS) consisted of a fraction with high contents of PEO that is soluble in PBS and a PEOT/PBT fraction that is insoluble at room temperature. From the different in vitro and in vivo degradation experiments, PEOT/ PBT degradation was slow and generates insoluble polymeric residues with high PBT contents which could be tolerated by the body.

Single and double fibers made of PLLA, PDO and PGCL have been prepared for the design of stents for in vitro study in PBS (pH 7.4) at $37^{\circ} \mathrm{C}$ [91]. At room temperature, PLLA was observed to possess a yield point with the highest tensile strength but the lowest ductility as compared with PDS and PGCL. Degradation studies of fibers indicated that PLLA undergoes slow degradation and can therefore preserve good mechanical properties after 24 weeks owing to low percentage loss in its mechanical strength as compared to PDS and PGCL. Although stents were observed to exhibit excellent initial radial compression strength, degradation studies showed that in vitro degradation resistance of PLLA was the best and hence, could support body conduits such as blood vessels for prolonged periods. The single-fiber design used for PDS and PGCL could have been responsible for the rapid loss of mechanical strength and using a double-fiber design can improve their degradation resistance.

Govaerr et al. [92] captured time-dependent failure behaviours of the following amorphous PLLA, a racemic co-polymer PDLLA and a 70:30 PLDLLA with glass transition temperatures $58^{\circ} \mathrm{C}, 42^{\circ} \mathrm{C}$ and $52^{\circ} \mathrm{C}$ respectively. Result shows that the yield stress of samples increases in the order: PLDLLA < PDLLA < PLLA. Results from yield-true strain rate relationship for the three PLA materials showed that their deformation kinetics (obtained from the slope) is identical. Steady state plastic flow of polymers was explained to occur when applied stress 
induces dynamic rearrangement of polymer molecular segments. This increased deformation rate that culminated in failure as soon as a localized plastic deformation zone was created. Premature failure of polymers is attributed to their time-dependent character achieved under load applications. Thus in predicting the long term performance of any polymeric material, knowledge of its instantaneous strength will not be enough. A single loading geometry is not enough to explain the strength of a polymeric material. Long-term performance of a degradable construct can in principle be estimated in early stages of design using some predictive models.

Leaching salt process was confirmed to be an alternative means in the processing of PCL and poly-D,L-lactic acid-co-glycolic acid of ratio 50/50 (PLGA50) scaffolds for cell culture in tissue engineering [93]. Dense and porous scaffolds are prepared via casting and melting compression processes. Prior to the production of porous scaffolds, inclusion of sodium citrate $\left(\mathrm{Na}_{3} \mathrm{C}_{6} \mathrm{H}_{5} \mathrm{O}_{7}\right.$. $)$ with particle sizes of 180 - $250 \mu$ m diameter acted as porogen (pore creator). Both samples were immersed in distilled water for 30 hours and evaluations by SEM, DSC and TGA were done. The results showed that leaching salt process does not alter the properties (morphology and thermal properties) of the scaffolds as the time for immersion of samples needed for the removal of salt in distilled water was observed to be insufficient. Determining work of adhesion between biodegradable polymers and hydroxyapatite (HA) via wetting experiments and surface tension measurements has been investigated by Neuendorf et al. [94]. The polymers used include: PLLA, PDLA, PDLLA, 75/25 PDLA/PGA, 50/ 50/50 PDLA/ PGA and PCL. Mechanical fracture strengths of the HA/polymer bond measured using four-point bend tests is observed to increase with decrease in contact angle and vice versa. There is reduction in bond strength of HA/ polymer interfaces on exposure to humid environments. With these results, using ceramic/polymer composites as load bearing, synthetic bone-like materials may not be feasible as their mechanical stability could be severely compromised during in vivo degradation.

Chen [95] investigated the degradation behavior of PLLA, PLGA and PCL sponges, having different glass transition temperature $\left(\mathrm{T}_{\mathrm{g}}\right)$ for structural change effect during degradation in PBS under $\mathrm{pH} 7.4$ at $37^{\circ} \mathrm{C}$. Analysis obtained from changes in average molecular weight $\left(\mathrm{M}_{\mathrm{n}}\right)$, weight loss, crystallinity $\left(\mathrm{X}_{\mathrm{c}}\right)$, melting temperature $\left(T_{m}\right), T_{g}$, and enthalpy revealed that PLGA sponges degrade most rapidly. Weight loss of the PLGA sponges reaches approximately $80 \%$ for 24 weeks of incubation. PLLA loses approximately $16 \%$ of its original weight after 26 weeks of incubation and PCL, less than 1\% after 26 weeks of incubation. Degradation of PLGA sponges was found to be dominated by autocatalyzed bulk degradation for the first 12 weeks with subsequent surface degradation. PLGA sponges showed physical ageing during incubation because its non-crystalline nature had a $\mathrm{T}_{\mathrm{g}}$ above but near the incubation temperature.

Two kinds of biodegradable polymer blends of PLA/PCL and polylactic acid/polycaprolactide/lysine triisocyanate (PLA/PCL/LTI), have been prepared and subjected to annealing to study its influence on their mechanical properties [96]. Fixed mixing ratio of PLA and PCL at 85:15 in weight fraction was used, with the inclusion of $1 \mathrm{wt} \%$ LTI content. The observed results revealed crystallization of the PLA phase by annealing at $100^{\circ} \mathrm{C}$ for 3 hours strengthens the structure of the PLA/PCL blend, resulting in increase of its bending modulus and strength. The significant decrease in the mode I fracture energy of PLA/PCL by annealing was attributed to the embrittlement of the PLA phase. In the case of PLA/PCL/LTI, the structural transformation due to polymerization by LTI addition and crystallization via annealing strengthened the microstructure which resulted to the dramatic improvement of the mode I fracture energy. Pins made of four tyrosine-derived polycarbonates-polyDTE carbonate, polyDTB carbonate, polyDTH carbonate, and polyDTiP carbonate have been incubated in PBS at $37^{\circ} \mathrm{C}, 50^{\circ} \mathrm{C}$, and $65^{\circ} \mathrm{C}$ for 36 months for hydrolytic degradation studies by Tangpasuthadol, et al. [97]. At the end of incubation, polyDTE carbonate and polyDTiP carbonate specimen remained transparent with few small cracks at the pin surface while pins of polyDTB carbonate and polyDTH carbonate bent and turned opaque after 3 months and two weeks, respectively. There was no mass loss observed during this period at $37^{\circ} \mathrm{C}$ unlike at $50^{\circ} \mathrm{C}$ and $65^{\circ} \mathrm{C}$, where mass loss was evident. Thus, it is reasonable to presume that loss of mass will also occur at $37^{\circ} \mathrm{C}$, after extensive backbone carbonate cleavage and pendent chain ester hydrolysis.

Russias et al. [98] worked on fabrication and mechanical properties of poly-L-lactic acid-hydroxyapatite (PLA/HA) composites produced by adding calcinated HA (hydroxyapatite) powders and HA whiskers to PLA. Immersing the composites in Hanks' Balanced Salt Solution, Young's modulus, bending strength and fracture toughness were discovered to deteriorate gradually. Composites with ceramic contents ranging between 70 and $85 \mathrm{wt} \%$ have mechanical properties that match reasonably those of human cortical bone. Degradation was more pronounced in samples with larger ceramic content due to the dissolution of the smaller amount of polymer between the ceramic particles. 


\section{Summary}

Although studies have shown that modifications in materials design can facilitate improved performance of implants, a careful study of the relationships among varying polymer components, their mechanical, chemical properties and degradation time need to be carried out. Thus, synchronization of polymer implant degradation with the replacement of natural tissues produced from cells in bone healing will facilitate adequate use of polymers in orthopedics.

\section{Conclusion}

In the reviewed literatures, synthetic biodegradable polymers and their blends have been attractive as tissue implantation devices owing to their biocompatibility and in particular, their controlled degradation kinetics in vivo and in vitro. It has always been a conventional perspective for researchers in materials science to tackle complications arising from the use of biodegradable polymers in orthopedics through the design of a new one. To achieve this, careful study of the properties and degradation kinetics of these synthetic polymers is advocated. In addition, more work needs to be carried out on the use of these ubiquitous natural polymers-starch and chitin, PLA with agro-wastes plant fibers in orthopedics as their usage will boost the commercialization of bone implants.

\section{References}

[1] Rodríguez, B., Romero, A., Soto, O. and Varorna, O. (2004) Biomaterials for Orthopaedics. Applications of Engineering Mechanics in Medicine, GED, 1-26.

[2] Seal, C.K., Vince, K. and Hodgson, M.A. (2009) Biodegradable Surgical Implants Based on Magnesium Alloys-A Review of Current Research. IOP Science, 4, 1-4.

[3] Hermawan, H., Ramdan, D. and Djuansjah, J.R.P. (2011) Metals for Biomedical Applications. InTech, 411-430. www.intechopen.com

[4] Hallab, N., Merritt, K. and Jacobs, P.J. (2006) Metal Sensitivity in Patients with Orthopaedic Implants. The Journal of Bone and Joint Surgery, 83-A, 427-436.

[5] Reifenrath, J., Bormann, D. and Lindenberg, A.M. (2011) Magnesium Alloys as Promising Degradable Implant Materials in Orthopaedic Research. InTech, 94-106. www.intechopen.com

[6] Rosson, J., Egan, J., Shearer, J. and Monro, P. (1991) Bone Weakness after the Removal of Plates and Screws. British Editorial Society of Joint and Bone Surgery, 72-B, 283-286.

[7] Rosson, J.W. and Shearer, J.R. (1991) Refracture after the Removal of Plates from the Fore Arm. The Journal of Bone and Joint Surgery, 73-B, 415-417.

[8] Abdul Razak, S.I., Sharif, N.F.A. and Abdul Rahman, W.A. (2012) Biodegradable Polymers and Their Bone Applications: A Review. International Journal of Basic and Applied Sciences, 12, 31-49.

[9] Marolt, D., Knezevic, K. and Novakovic, G.V. (2010) Bone Tissue Engineering with Human Stem Cells. Stem Cell Research and Therapy, 1, 10.

[10] Elias, N., Lima, J.H.C., Valiev, R. and Meyers, M.A. (2008) Biomedical Applications of Titanium and Its Alloys. JOM, 60, 46-49.

[11] Dan, C.N. (2008) Titanium and Titanium Alloys for Biomedical and Industry Applications. WESIC 08, Bucharest, 25-26 September, 128-132.

[12] Middleton, J.C. and Tipton, A.J. (2000) Synthetic Biodegradable Polymers as Orthopaedic Devices. Biomaterials, 21, 2335-2346. http://dx.doi.org/10.1016/S0142-9612(00)00101-0

[13] Jacobs, J.J., Gilbert, J.L. and Urban, R.M. (1998) Current Concepts Review Corrosion of Metal Orthopaedic Implants. The Journal of Bone and Joint Surgery, 80, 268-282.

[14] Hansen, D.C. (2008) Metal Corrosion in the Human Body: The Ultimate Bio-Corrosion Scenario. The Electrochemical Society Interface, 17, 31-34.

[15] Geringer, J., Forest, B. and Combcade, P. (2005) Fretting-Corrosion of Materials Used as Orthopaedic Implants. Wear, 259, 943-951. http://dx.doi.org/10.1016/j.wear.2004.11.027

[16] Mitchell, A. and Shrotriya, P. (2007) Onset of Nanoscale Wear of Metallic Implant Materials: Influence of Surface Residual Stresses and Contact Loads. Wear, 263, 1117-1123. http://dx.doi.org/10.1016/j.wear.2007.01.068

[17] Farrar, D. (2005) Bioresorbable Polymers in Orthopaedics. Medical Device Manufacturing and Technology, 1-4. 
[18] Gunatillake, P.A. and Adhikari, R. (2003) Biodegradable Synthetic Polymers for Tissue Engineering. European Cells and Materials, 5, 1-16.

[19] Buddy, D.R., Allan, S.H., Fredrick, J.S. and Jack, E.S. (1996) Biomaterials Science. Academic Press, Waltham, 1-497.

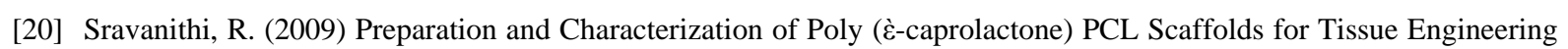
Scaffolds. Department of Biotechnology and Medical Engineering, 1-59.

[21] Peltoniemi, H. (2000) Biocompatibility and Fixation Properties of Absorbable Mini Plates and Screws in Calvarium. Department of Surgery, University of Helsinki, Helsinki, 1-83.

[22] Puleo, D.A. and Nanci, A. (1999) Understanding and Controlling the Bone Implant Interface. Biomaterials, 20, 23112321. http://dx.doi.org/10.1016/S0142-9612(99)00160-X

[23] Bibber, D. (2009) Micromoulding with Resorbable Materials. The Magazine for the Global Micro Manufacturing Technology Community.

[24] Dunne, M., Corrigan, O.I. and Ramtoola, Z. (2000) Influence of Particle Size and Dissolution Conditions on the Degradation Properties of Polylactide-co-glycolide Particles. Biomaterials, 21, 1659-1668. http://dx.doi.org/10.1016/S0142-9612(00)00040-5

[25] Thomson, R.C., Mikos, A.G., Beahm, E., Lemon, J.C., Satterfield, W.C., Aufdemorte, T.B. and Miller, M.J. (1999) Guided Tissue Fabrication from Periosteum Using Preformed Biodegradable Polymer Scaffolds. Biomaterials, 20, 2007-2018.

[26] MAST Biosurgery Inc. (2006) MAST Biosurgery Resorbable Technology: An Overview. 1-6. www.mastbio.com

[27] Ciambelli, G.S., Perez, M.O., Siqueira, G.V., Candellaa, M.A., Mottaa, A.C., Duartea, M.A.T., Rincon, M.C.A. and Duek, E.A.R. (2013) Characterization of Poly (L-co-D,L lactic Acid) and a Study of Polymer-Tissue Interaction in Subcutaneous Implants in Wistar Rats. Materials Research, 16, 28-37. http://dx.doi.org/10.1590/S1516-14392012005000146

[28] Jeong, S.I., Kim, B.S., Kang, S.W., Kwon, J.H., Lee, Y.M., Kim, S.H. and Kim, Y.H. (2004) In Vivo Biocompatibilty and Degradation Behavior of Elastic Poly(L-lactide-co-e-Caprolactone) Scaffolds. Biomaterials, 25, 5939-5946. http://dx.doi.org/10.1016/j.biomaterials.2004.01.057

[29] Manzi, K. (2009) A Study of Tensile Degradation of Bioresorbable Materials Used in Internal Fixation of Bones. 1-12. http://www.scientiareview.org/pdfs/75.pdf

[30] Xiao, L., Wang, B., Yang, G. and Gauthier, M. (2012) Poly (Lactic Acid)-Based Biomaterials: Synthesis, Modification and Applications. In: Ghista, D.N., Ed., Biomedical Science, Engineering and Technology, 247-282.

[31] Salgado, A.J., Coutinho, O.P., Reis, R.L. and Davies, J.E. (2006) In Vivo Response to Starch-Based Scaffolds Designed for Bone Tissue Engineering Applications. Journal of Biomaterials Research, 80, 983-989.

[32] Silva, T.H., Alves, A., Ferreira, B.M., Oliveira, J.M., Reys, L.L., Ferreira, R.J.F., Sousa, R.A., Silva, S.S., Mano, J.F. and Reis, R.L. (2012) Materials of Marine Origin: A Review on Polymers and Ceramics of Biomedical Interest. International Materials Reviews, 1-32. http://repositorium.sdum.uminho.pt/bitstream/1822/21720/1/imr200.pdf

[33] Duta, P.K., Duta, J. and Tripathi, V.S. (2004) Chitin and Chitosan: Chemistry Properties and Applications. Journal of Scientific and Industrial Research, 63, 20-31.

[34] Isa, M.T., Ameh, A.O., Gabriel, A.O. and Adamma, K.K. (2012) Extraction and Characterization of Chitin from Nigerian Source. Leonardo Electronic Journal of Practices and Technologies, 21, 73-81.

[35] Struszczyk, M.H. (2006) Global Requirements for Medical Applications of Chitin and Its Derivatives. Polish Chitin Society, Monograph XI, 95-102. http://ptchit.lodz.pl/PTChit/11_12.pdf

[36] Ge, Z., Baguenard, S., Lim, L.Y., Wee, A. and Khor, E. (2004) Hydroxyapatite-Chitin Materials as Potential Tissue Engineered Bone Substitutes. Biomaterials, 25, 1049-1058. http://dx.doi.org/10.1016/S0142-9612(03)00612-4

[37] Shigemasa, Y. and Minami, S. (1996) Application of Chitin and Chitosan for Biomaterial. Biotechnology and Genetic Engineering Reviews, 13, 383-420. http://dx.doi.org/10.1080/02648725.1996.10647935

[38] Kohr, E. and Lim, L.Y. (2003) Implantable Application of Chitin and Chitosan. Biomaterials, 24, 2339-2349. http://dx.doi.org/10.1016/S0142-9612(03)00026-7

[39] Vieira, A.C., Vieira, J.C., Ferra, J.M., Magalhães, F.D., Guedes, R.M. and Marques, A.T. (2011) Mechanical Study of PLA-PCL Fibers during in Vitro Degradation. Journal of Mechanical Behaviour of Biomedical Materials, 4, 451-460. http://dx.doi.org/10.1016/j.jmbbm.2010.12.006

[40] Lyu, S. and Unterecker, D. (2009) Degradability of Polymers for Implantable Biomedical Devices. International Journal of Molecular Sciences, 10, 4033-4065. http://dx.doi.org/10.3390/ijms10094033

[41] Schliecker, G., Schmidt, C., Fuchs, S. and Kisse, T. (2003) Characterization of a Homologous Series of D,L-Lactic Acid Oligomers; a Mechanistic Study on the Degradation Kinetics in Vitro. Biomaterials, 24, 3835-3844. 
http://dx.doi.org/10.1016/S0142-9612(03)00243-6

[42] Santoven, A., Lorenzo, C.A., Concheiro, A., Llabres, M. and Farin, J.B. (2004) Rheological Properties of PLGA Film-Based Implants: Correlation with Polymer Degradation and SPf66 Antimalaric Synthetic Peptide Release. Biomaterials, 25, 925-931. http://dx.doi.org/10.1016/S0142-9612(03)00592-1

[43] Vieira, A.C., Vieira, J.C., Guedes, R.M. and Marques, A.T. (2010) Experimental Degradation Characterization of PLA-PCL, PGA-PCL, PDO and PGA Fibers. Materials Science Forum, 636-637, 825-832. http://dx.doi.org/10.4028/www.scientific.net/MSF.636-637.825

[44] Hasirc1, V., Lewandrowski, K., Gresser, J.D., Wise, D.L. and Trantolo, D.J. (2001) Versatility of Biodegradable Biopolymers: Degradability and an in Vivo Application. Journal of Biotechnology, 86, 135-150. http://dx.doi.org/10.1016/S0168-1656(00)00409-0

[45] Mi, F.L., Lin, Y.M., Wu, Y.B., Shyu, S.S. and Tsai, Y.H. (2002) Chitin/PLGA Blend Microspheres as a Biodegradable Drug-Delivery System: Phase-Separation, Degradation and Release Behavior. Biomaterials, 23, 3257-3267. http://dx.doi.org/10.1016/S0142-9612(02)00084-4

[46] Kumta, S.M., Spinner, R. and Cleung, P. (1992) Absorbable Intramedullary Implants for Hand Fractures. Journal of Bone and Joint Surgery, 74, 563-566.

[47] Glarner, M. and Gogolewski, S. (2004) Degradation in Vitro of New Bioresorbable Terpolymers of Lactides. European Cells and Materials, 7, 36.

[48] Tangpasuthadol, V., Pendharkar, S.M. and Kohn, J. (2000) Hydrolytic Degradation of Tyrosine-Derived Polycarbonates, a Class of New Biomaterials. Part I: Study of Model Compounds. Biomaterials, 21, 2371-2378. http://dx.doi.org/10.1016/S0142-9612(00)00104-6

[49] Duek, E.A.R., Zavaglia, C.A.C. and Belangero, W.D. (1999) In Vitro Study of Poly(Lactic Acid) Pin Degradation. Polymer, 40, 6465-6473. http://dx.doi.org/10.1016/S0032-3861(98)00846-5

[50] Wu, X.S. and Wang, N. (2001) Synthesis, Characterization, Biodegradation, and Drug Delivery Application of Biodegradable Lactic/Glycolic Acid Polymers. Part II: Biodegradation. Journal of Biomaterials Science, Polymer Edition, 12, 21-34. http://dx.doi.org/10.1163/156856201744425

[51] Zignani, M., Minh, T.L., Einmahl, S., Tabatabay, C., Heller, J., Anderson, J.M. and Gurny, R. (2000) Improved Biocompatibility of a Viscous Bioerodible Poly(Ortho Ester) by Controlling the Environmental pH during Degradation. Biomaterials, 21, 1773-1778. http://dx.doi.org/10.1016/S0142-9612(00)00065-X

[52] Ali, S.A.M., Doherty, P.J. and Williams, D.F. (1993) Mechanisms of Polymer Degradation Implantable,2,Poly-D, L-Lactic Acid. Journal of Biomedical Materials Research, 27, 1409-1418. http://dx.doi.org/10.1002/jbm.820271108

[53] Schmidmaier, G., Wildemann, B., Stemberger, A., Haas, N.P. and Raschke, M. (2001) Biodegradable Poly(D,L-Lactide) Coating of Implants for Continuous Release of Growth Factors. Journal of Biomedical Materials Research, 58, 449455.

[54] Park, A. and Cima, L.G. (1996) In Vitro Cell Response to Differences in Poly-L-lactide Crystallinity. Journal of Biomedical Materials Research, 31, 117-130. http://dx.doi.org/10.1002/jbm.1996.820310103

[55] Barbanti, S.H., Zavaglia, C.A.C., Santos Jr., A. and Duek, E.A.R. (2006) Poly(L-Lactic Acid) Scaffold: In Vitro Degradation in Alkaline Medium, Phosphate Buffer Solution and Osteoblast Morphology. Revista CENIC. Ciencias Biológicas, 37, 182-187.

[56] Fu, B.X. and Hsiao, B.S. (2003) A Study of Structure and Property cChanges of Biodegradable Polyglycolide and Polyglycolide-co-Lactide Fibres during Processing and in Vitro Degradation. Chinese Journal of Polymer Science, 21, 159-167.

[57] Sultana, N. and Abdul Kadir, M.R. (2011) Study of in Vitro Degradation of Biodegradable Polymer Based Thin Films and Tissue Engineering Scaffolds. African Journal of Biotechnology, 10, 18709-18715.

[58] Grizzi, I., Garreau, H., Li, S. and Vert, M. (1995) Hydrolytic Degradation of Devices Based on Poly[m-Lactic Acid) Size Dependence. Biomaterials, 16, 305-311. http://dx.doi.org/10.1016/0142-9612(95)93258-F

[59] Glauser, A.C.R., Rose, J., Farrar, D. and Cameron, R.E. (2005) A Degradation Study of PLLA Containing Lauric Acid. Biomaterials, 26, 2415-2422. http://dx.doi.org/10.1016/j.biomaterials.2004.07.067

[60] Cristian, A.N., Grigorescu, M.A. and Gabor, R.A. (2008) An Investigation of Thermal Degradation of Poly(Lactic Acid). Engineering Letter, 16, 1-4.

[61] Nugroho, P. and Mitomo, H. (2008) Study of Biodegradation and Improvement of Heat Stability of Poly(Lactic Acid) by Irradiation at High Temperature. Malaysian Polymer Journal, 31, 27-37.

[62] Perez, J.G., Velazquez-Infante, J.C., Franco-Urquiza, E., Pages, P., Carrasco, F., Santana, O.O. and Maspoch, M.L. (2011) Fracture Behavior of Quenched Poly(Lactic Acid). eXPRESS Polymer Letters, 5, 82-91.

[63] Park, S.D., Todo, M., Arakawa, K. and Koganemaru, M. (2006) Effect of Crystallinity and Loading-Rate on Mode I 
Fracture Behavior of Poly(Lactic Acid). Polymer, 47, 1357-1363. http://dx.doi.org/10.1016/j.polymer.2005.12.046

[64] Elst, M., Dijkema, A.R.A., Klein, C.P.A.T., Patka, P. and Haarman, H.J.Th.M. (1995) Tissue Reaction on PLLA Versus Stainless Steel Interlocking Nails for Fracture Fixation: An Animal Study. Biomaterials, 16, 103-106. http://dx.doi.org/10.1016/0142-9612(95)98270-O

[65] Viljanen, J., Kinnunen, J., Bondestam, S., Majola, A., Rokkanen, P. and Tormala, P. (1995) Bone Changes after Experimental Osteotomies Fixed with Absorbable Self-Reinforced Poly-L-Lactide Screws or Metallic Screws Studied by Plain Radiographs, Quantitative Computed Tomography and Magnetic Resonance Imaging. Biomaterials, 16, 13531358. http://dx.doi.org/10.1016/0142-9612(95)91052-Z

[66] Bostman, O., Viljanen, J., Salminen, S. and Pihlajamaki, H. (2000) Response of Articular Cartilage and Sub-Chondral Bone to Internal Fixation Devices Made of Poly-L-Lactide: A Histomorphometric and Micro Radiographic Study on Rabbits. Biomaterials, 21, 2553-2560. http://dx.doi.org/10.1016/S0142-9612(00)00123-X

[67] Pekkanuutinen, K., Rreinikainen, C.C.R. and Törmälä, P. (2003) Mechanical Properties and in Vitro Degradation of Bioabsorbable Self-Expanding Braided Stents. Journal of Biomaterials Science. Polymer Edition, 14, 255-266. http://dx.doi.org/10.1163/156856203763572707

[68] Prokop, A., Jubel, A., Helling, H.J., Eibach, T., Peters, C., Baldus, S.E. and Rehm, K.E. (2004) Soft Tissue Reactions of Different Biodegradable Polylactide Implants. Biomaterials, 25, 259-267. http://dx.doi.org/10.1016/S0142-9612(03)00496-4

[69] Schantz, J.T., Lim, T.C., Ning, C., Teoh, S.H., Tan, K.C., Wang, S.C. and Hutmacher, D.W. (2006) Cranioplasty after Trephination Using a Novel Biodegradable Burr Hole Cover: Technical Case Report. Neurosurgery, 58, ONS-E176.

[70] Hafeman, A.E., Zienkiewicz, K.J., Zachman, A.L., Sung, H.J., Nanney, L.B., Davidson, J.M. and Guelcher, S.A. (2011) Characterization of the Degradation Mechanisms of Lysine-Derived Aliphatic Poly(Ester Urethane) Scaffolds. Biomaterials, 32, 419-429. http://dx.doi.org/10.1016/j.biomaterials.2010.08.108

[71] Farrar, D.F. and Gillson, R.K. (2002) Hydrolytic Degradation of Polyglyconate B: The Relationship between Degradation Time, Strength and Molecular Weight. Biomaterials, 23, 3905-3912. http://dx.doi.org/10.1016/S0142-9612(02)00140-0

[72] Broz, M.E., VanderHart, D.L. and Washburn, N.R. (2003) Structure and Mechanical Properties of Poly-D,L-Lactic Acid/poly- $\varepsilon$-Caprolactone Blends. Biomaterials, 24, 4181-4190. http://dx.doi.org/10.1016/S0142-9612(03)00314-4

[73] Todo, M., Park, S.D., Takayama, T. and Arakawa, K. (2007) Fracture Micromechanisms of Bioabsorbable PLLA/PCL Polymer Blends. Engineering Fracture Mechanics, 74, 1872-1883. http://dx.doi.org/10.1016/j.engfracmech.2006.05.021

[74] Salgado, C.L., Sanchez, E.M.S., Zavaglia, C.A.C. and Granja, P.L. (2011) Biocompatibility and Biodegradation of Polycaprolactone-Sebacic Acid Blended Gels. Journal of Biomedical Materials Research, 100, 243-251.

[75] Holy, C.E., Dang, S.M., Davies, J.E. and Shoichet, M.S. (1999) In Vitro Degradation of a Novel Poly(Lactide-co-Glycolide) 75/25 Foam. Biomaterials, 20, 1177-1185. http://dx.doi.org/10.1016/S0142-9612(98)00256-7

[76] Elst, M., Klein, C.P.A.T., Hogervorst, J.M.B., Patk, P. and Haarman, H.J.Th.M. (1999) Bone Tissue Response to Biodegradable Polymers Used for Intra Medullary Fracture Fixation: A Long-Term in Vivo Study in Sheep Femora. Biomaterials, 20, 121-128.

[77] Lin, Y.S., Feng, C.K., Ye, S.B., Lin, Y.J., Chen, C.F., Liao, C.J., Chang, K.Y. and Tsay, R.Y. (2001) An in Vivo Study on the Biocompatibility of a Bioresorbable Poly(L-Lactide-co-Glycolide) Pin for Bone Fixation. Journal of Medical and Biological Engineering, 21, 233-242.

[78] Bruggeman, J.P., Bruin, B.J., Bettinger, C.J. and Langer, R. (2008) Biodegradable Polypolyolsebacate Polymers. Biomaterials, 29, 4726-4735. http://dx.doi.org/10.1016/j.biomaterials.2008.08.037

[79] Chłopek, J., Chochół, A.M. and Szaraniec, B. (2010) The Influence of the Environment on the Degradation of Polylactides and Their Composites. Journal of Achievements in Materials and Manufacturing Engineering, 43, 72-79.

[80] Smit, T.H., Engels, T.A.P., Wuisman, P.I.J.M. and Govaerr, L.E. (2008) Time-Dependent Mechanical Strength of 70/30 Poly(L,DL-Lactide). SPINE, 33, 14-18. http://dx.doi.org/10.1097/BRS.0b013e31815e39df

[81] Barauna, G., Huberb, D.C.C. and Duek, E.A.R. (2013) In Vitro Degradation of Poly-L-co-D,L-Lactic Acid Membranes. Materials Research, 16, 221-226. http://dx.doi.org/10.1590/S1516-14392012005000154

[82] Timmer, M.D., Ambrose, C.G. and Mikos, A.G. (2003) In Vitro Degradation of Polymeric Networks of Poly(Propylene Fumarate) and the Crosslinking Macromere Poly(Propylene Fumarate)-Diacrylate. Biomaterials, 24, 571-577. http://dx.doi.org/10.1016/S0142-9612(02)00368-X

[83] Todo, M., Harada, A. and Tsuji, H. (2007) Fracture Characterization of Biodegradable PLLA Polymer Blends. 16th International Conference on Composite Materials, Kyoto, 8-13 July, 1-6. 
[84] Schakenraad, J.M., Nieuwenhuis, P. and Molenaar, I. (1989) In Vivo and in Vitro Degradation of Glycine/DL-Lactic Acid Copolymers. Journal of Biomedical Materials Research, 23, 1271-1288. http://dx.doi.org/10.1002/jbm.820231105

[85] Riley, S.L., Okun, L.E., Prado, G., Applegate, M.A. and Ratclie, A. (1999) Human Articular Chondrocyte Adhesion and Proliferation on Synthetic Biodegradable Polymer Films. Biomaterials, 20, 2245-2256.

[86] Kiremitci, M. and Deniz, G. (1999) Synthesis, Characterization and in Vitro Degradation of Poly(DL-Lactide)/ Poly(DL-Lactide-co-Glycolide) Films. Turkish Journal of Chemistry, 23, 153-161.

[87] Nordstrom, P., Pohjonen, T., Tormala, P. and Rokkanen, P. (2001) Shear-Load Carrying Capacity of Cancellous Bone after Implantation of Self-Reinforced Polyglycolic Acid and Poly-L-Lactic Acid Pins: Experimental Study on Rats. Biomaterials, 22, 2557-2561. http://dx.doi.org/10.1016/S0142-9612(00)00446-4

[88] Freier, T., Kunze, C., Nischan, C., Kramer, S., Sternberg, K., Saß, K., Hopt, U.T. and Schmit, K.P. (2002) In Vitro and in Vivo Degradation Studies for Development of a Biodegradable Patch Based on Poly(3-Hydroxybutyrate). Biomaterials, 23, 2649-2657.

[89] Sung, H.J., Meredith, C., Johnson, C. and Galis, Z.C. (2004) The Effect of Scaffold Degradation Rate on Three-Dimensional Cell Growth and Angiogenesis. Biomaterials, 25, 5735-5742. http://dx.doi.org/10.1016/j.biomaterials.2004.01.066

[90] Deschamps, A.A., Van Apeldoorn, A.A., Hayenc, H., De Bruijn, J.D., Karst, U., Grijpma, D.W. and Feijen, J. (2004) In Vivo and in Vitro Degradation of Poly(Ether Ester) Block Copolymers Based on Poly(Ethylene Glycol) and Poly(Butylene Terephthalate). Biomaterials, 25, 247-258. http://dx.doi.org/10.1016/S0142-9612(03)00495-2

[91] Zilberman, M., Nelson, K.D. and Eberhart, R.C. (2005) Mechanical Properties and in Vitro Degradation of Bioresorbable Fibers and Expandable Fiber-Based Stents. Journal of Biomedical Materials Research, 74, 792-799.

[92] Govaerr, L.E., Engels, T.A.P., Söntjens, S.H.M. and Smit, T.H. (2010) Time-Dependent Failure in Load-Bearing Polymers. A Potential Hazard in Structural Applications of Polylactides. Journal of Materials Science: Materials in Medicine, 21, 871-878.

[93] Barbanti, S.H., Zavaglia, C.A.C. and Duek, E.A.R. (2008) Effect of Salt Leaching on PCL and PLGA(50/50) Resorbable Scaffolds. Materials Research, 11, 75-80. http://dx.doi.org/10.1590/S1516-14392008000100014

[94] Neuendorf, R.E., Saiz, E., Tomsia, A.P. and Ritchie, R.O. (2008) Adhesion between Biodegradable Polymers and Hydroxyapatite: Relevance to Synthetic Bone-Like Materials and Tissue Engineering Scaffolds. Acta Biomaterialia, 4, 1288-1296. http://dx.doi.org/10.1016/j.actbio.2008.04.006

[95] Chen, G. (2010) Degradation Behavior of Aliphatic Biodegradable Polyester. Society of Plastics Engineers, 1-2. http://4spepro.org/pdf/003047/003047.pdf

[96] Takayama, T., Todo, M. and Tsuj, H. (2011) Effect of Annealing on the Mechanical Properties of PLA/PCL and PLA/PCL/LTI Polymer Blends. Journal of the Mechanical Behavior of Biomedical Materials, 4, 255-260. http://dx.doi.org/10.1016/j.jmbbm.2010.10.003

[97] Tangpasuthadol, V., Pendharkar, S.M., Peter, R.M. and Kohn, J. (2000) Hydrolytic Degradation of Tyrosine-Derived Polycarbonates, a Class of New Biomaterials. Part II: 3-yr Study of Polymeric Devices. Biomaterials, 21, 2379-2387. http://dx.doi.org/10.1016/S0142-9612(00)00105-8

[98] Russias, J., Saiz, E., Nalla, R.K., Gryn, K., Ritchie, R.O. and Tomsia, A.P. (2006) Fabrication and Mechanical Properties of PLA/HA Composites: A Study of in Vitro Degradation. Materials Sciences and Engineering: C, 26, 1289-1295. http://dx.doi.org/10.1016/j.msec.2005.08.004 